\title{
A Re-examination on Features in Regression Based Approach to Auto- matic MT Evaluation
}

\author{
Shuqi Sun, Yin Chen and Jufeng Li \\ School of Computer Science and Technology \\ Harbin Institute of Technology, Harbin, China \\ \{sqsun, chenyin, jfli\}@mtlab.hit.edu.cn
}

\begin{abstract}
Machine learning methods have been extensively employed in developing MT evaluation metrics and several studies show that it can help to achieve a better correlation with human assessments. Adopting the regression SVM framework, this paper discusses the linguistic motivated feature formulation strategy. We argue that "blind" combination of available features does not yield a general metrics with high correlation rate with human assessments. Instead, certain simple intuitive features serve better in establishing the regression SVM evaluation model. With six features selected, we show evidences to support our view through a few experiments in this paper.
\end{abstract}

\section{Introduction}

The automatic evaluation of machine translation (MT) system has become a hot research issue in MT circle. Compared with the huge amount of manpower cost and time cost of human evaluation, the automatic evaluations have lower cost and reusability. Although the automatic evaluation metrics have succeeded in the system level, there are still on-going investigations to get reference translation better (Russo-Lassner et al., 2005) or to deal with sub-document level evaluation (Kulesza et al., 2004; Leusch et al, 2006).

$\mathrm{N}$-grams' co-occurrence based metrics such as BLEU and NIST can reach a fairly good correlation with human judgments, but due to their consideration for the capability of generalization across multiple languages, they discard the inherent linguistic knowledge of the sentence evaluated.
Actually, for a certain target language, one could exploit this knowledge to help us developing a more "human-like" metric. Giménez and Márquez (2007) showed that compared with metrics limited in lexical dimension, metrics integrating deep linguistic information will be more reliable.

The introduction of machine learning methods aimed at the improvement of MT evaluation metrics' precision is a recent trend. Corston-Oliver et al. (2001) treated the evaluation of MT outputs as classification problem between human translation and machine translation. Kulesza et al. (2004) proposed a SVM classifier based on confidence score, which takes the distance between feature vector and the decision surface as the measure of the MT system's output. Joshua S. Albrecht et al. (2007) adopted regression SVM to improve the evaluation metric.

In the rest of this paper, we will first discuss some pitfalls of the n-gram based metrics such as BLEU and NIST, together with the intuition that factors from the linguist knowledge can be used to evaluate MT system's outputs. Then, we will propose a MT evaluation metric based on SVM regression using information from various linguistic levels (lexical level, phrase level, syntax level and sentence-level) as features. Finally, from empirical studies, we will show that this metric, with less simple linguistic motivated features, will result in a better correlation with human judgments than previous regression-based methods.

\section{N-gram Based vs Linguistic Motivated Metrics}

$\mathrm{N}$-gram co-occurrence based metrics is the main trend of MT evaluation. The basic idea is to compute the similarity between MT system output and 
several human reference translations through the co-occurrence of n-grams. BLEU (Papineni et al., 2002) is one of the most popular automatic evaluation metrics currently used. Although with a good correlation with human judgment, it still has some defects:

- BLEU considers precision regardless of recall. To avoid a low recall, BLEU introduces a brevity penalty factor, but this is only an approximation.

- Though BLEU makes use of high order ngrams to assess the fluency of a sentence, it does not exploit information from inherent structures of a sentence.

- BLEU is a "perfect matching only" metric. This is a serious problem. Although it can be alleviated by adding more human reference translations, there may be still a number of informative words that will be labeled as "unmatched".

- BLEU lacks models determining each ngram's own contribution to the meaning of the sentence. Correct translations of the headwords which express should be attached more importance to than that of accessory words e.g.

- While computing geometric average of precisions from unigram to $n$-gram, if a certain precision is zero, the whole score will be zero.

In the evaluation task of a MT system with certain target language, the intuition is that we can fully exploit linguistic information, making the evaluation progress more "human-like" while leaving the capability of generalization across multiple languages (just the case that BLEU considers) out of account.

Following this intuition, from the plentiful linguist information, we take the following factors in to consideration:

- Content words are important to the semantic meaning of a sentence. A better translation will include more substantives translated from the source sentence than worse ones. In a similar way, a machine translation should be considered a better one, if more content words in human reference translations are included in it.

- At the phrase level, the situation above remains the same, and what is more, real phrases are used to measure the quality of the machine translations instead of merely using n-grams which are of little semantic information.

- In addition, the length of translation is usually in good proportion to the source language. We believe that a human reference translation sentence has a moderate byte-length ratio to the source sentence. So a machine translation will be depreciated if it has a ratio considerably different from the ratio calculated from reference sentences.

- Finally, a good translation must be a "wellformed" sentence, which usually brings a high probability score in language models, e.g. n-gram model.

In the next section, using regression SVM, we will build a MT evaluation metric for ChineseEnglish translation with features selected from above aspects.

\section{A Regression SVM Approach Based on Linguistic Motivated Features}

Introducing machine learning methods to establish MT evaluation metric is a recent trend. Provided that we could get many factors of human judgments, machine learning will be a good method to combine these factors together. As proved in the recent literature, learning from regression is of a better quality than from classifier (Albrecht and Hwa, 2007; Russo-Lassner et al., 2005; Quirk, 2004). In this paper, we choose regression support vector machine (SVM) as the learning model.

\subsection{Learning from human assessment data}

The machine translated sentences for model training are provided with human assessment data score together with several human references. Each sentence is treated as a training example. We extract feature vectors from training examples, and human assessment score will act as the output of the target function. The regression SVM will generate an approximated function which maps multidimensional feature vectors to a continuous real value with a minimal error rate according to a loss function. This value is the result of the evaluation process.

Figure 1 shows our general framework for regression based learning, in which we train the SVM with a number of sentences $\boldsymbol{x}_{1}, \boldsymbol{x}_{2}, \ldots$ with human assessment scores $y_{1}, y_{2}, \ldots$ and use the trained model to evaluate an test sentence $\boldsymbol{x}$ with feature vector $\left(f_{1}, f_{2}, \ldots, f_{n}\right)$. To determine which indicators of a sentence are chosen as features is research in progress, but we contend that "the more features, the better quality" is not always true. Large feature sets require more computation cost, though maybe result in a metric with a better corre- 
lation with human judgments, it can also be achieved by introducing a much smaller feature set. Moreover, features may conflict with each others, and bring down the performance of the metric. We will show this in the next section, using less than 10 features stated in section 3.2. Some details of the implementation will also be described.

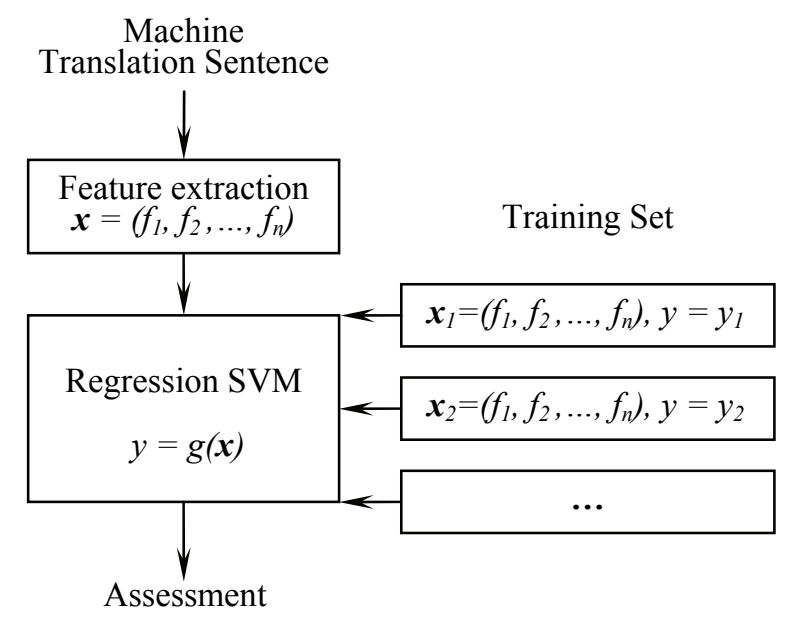

Figure 1: SVM based model of automatic MT evaluation metric

\subsection{Feature selection}

A great deal of information can be extracted from the MT systems' output using linguistic knowledge. Some of them can be very informative while easy to obtain.

As considered in section 2, we choose factors from lexical level, phrase level, syntax level and sentence-level as features to train the SVM.

- Features based on translation quality of content words

The motivation is that content words are carrying more important information of a sentence compared with function words. In this paper, content words include nouns, verbs, adjectives, adverbials, pronouns and cardinal numerals. The corresponding features are the precision of content words defined in Eq. 1 and the recall defined in Eq. 2 where ref means reference translation.

$$
\begin{aligned}
& \text { precision }_{\text {con }}(t)= \\
& \frac{\# \text { correctly_translated_cons_in_t } t}{\# \text { cons_in_t }}
\end{aligned}
$$

$\operatorname{recall}_{\text {con }}(t)=$

$$
\frac{\# \text { cons_in_ref_correctly_translated_in_t } t}{\text { \#cons_in_the_ref }}
$$

- Features based on cognate words matching
English words have plenty of morphological changes. So if a machine translation sentence shares with a human reference sentence some cognates, it contains at least some basic information correct. And if we look at it in another way, words that do not match in the original text maybe match after morphological reduction. Thus, differences between poor translations will be revealed. Similarly, we here define the content word precision and recall after morphological reduction in Eq. 3 and Eq. 4 where $m r$ cons means content words after morphological reduction:

$$
\begin{aligned}
& \text { precision }_{m r_{-} \text {con }}(t)= \\
& \frac{\# \text { correctly_translated_mr_cons_in_t } t}{\# m r_{-} \text {cons_in_t }} \\
& \text { recall }_{m r_{-} \text {con }}(t)= \\
& \frac{\# m r_{-} \text {cons_in_ref_correctly_translated_in_t } t}{\# m r_{-} \text {cons_in_the_ref }}
\end{aligned}
$$

- Features based on translation quality of phrases

Phrases are baring the weight of semantic information more than words. In manual evaluation, or rather, in a human's mind, phrases are paid special attention to. Here we parse every sentence ${ }^{1}$ and extract several types of phrases, then, compute the precision and recall of each type of phrase according to Eq. 5 and Eq. $6^{2}$ :

$$
\begin{aligned}
& \text { precision }_{p h r}(t)= \\
& \frac{\text { \#correctly_translated_phrs_in_t } t}{\# \text { phrs_in_t }} \\
& \text { recall }_{p h r}(t)= \\
& \frac{\# p h r_{-} i n_{-} r e f \_c o r r e c t l y \_t r a n s l a t e d_{-} i n_{-} t}{\# p h r_{-} i n_{-} t h e_{-} r e f}
\end{aligned}
$$

In practice, we found that if we compute these two indicators by matching phrases caseinsensitive, we will receive a metric with higher performance. We speculate that by doing this the difference between poor translations is revealed just like morphological reduction.

- Features based on byte-length ratio

Gale and Church (1991) noted that he bytelength ratio of target sentence to source sentence is normally distributed. We employ this observation by computing the ratio of reference sentences to

\footnotetext{
${ }^{1}$ The parser we used is proposed by Michael Collins in Collins (1999).

${ }^{2}$ Only precision and recall of NP are used so far. Other types of phrase will be added in future study.
} 
source sentences, and then calculating the mean $c$ and variance $s$ of this ratio. So if we take the ratio $r$ as a random variable, $(r-c) / s$ has a normal distribution with mean 0 and variance 1 . Then we compute the same ratio of machine translation sentence to source sentence, and take the output of p-norm function as a feature:

$$
f(t)=P_{\text {norm }}\left(\frac{\text { lenght_of_t } t \text { length_of_s } s r c-c}{s}\right)
$$

- Features based on parse score

The usual practice to model the "wellformedness" of a sentence is to employ the n-gram language model or compute the syntactic structure similarity (Liu and Gildea 2005). However, the language model is widely adopted in MT, resulting less discrimination power. And the present parser is still not satisfactory, leading much noise in parse structure matching.

To avoid these pitfalls in using LM and parser, here we notice that the score of a parse by the parser also reflects the quality of a sentence. It may be regarded as a syntactic based language model score as well as an approximate representation of parse structure. Here we introduce the feature based on parser's score as:

$$
\begin{aligned}
\text { paser_score }(t) & = \\
& -\frac{100}{\text { mark_of_t_given_by_parser }}
\end{aligned}
$$

\section{Experiments}

We use SVM-Light (Joachims 1999) to train our learning models. Our main dataset is NIST's 2003 Chinese MT evaluations. There are $6 \times 919=5514$ sentences generated by six systems together with human assessment data which contains a fluency score and adequacy score marked by two human judges. Because there is bias in the distributions of the two judges' assessment, we normalize the scores following Blatz et al. (2003). The normalized score is the average of the sum of the normalized fluency score and the normalized adequacy score.

To determine the quality of a metric, we use Spearman rank correlation coefficient which is distribution-independent between the score given to the evaluative data and human assessment data. The Spearman coefficient is a real number ranging from -1 to +1 , indicating perfect negative correlations or perfect positive correlations. We take the correlation rates of the metrics reported in Albrecht and Hwa (2007) and a standard automatic metric BLEU as a baseline comparison.

Among the features described in section 3.2, we finally adopted 6 features:

- Content words precision and recall after morphological reduction defined in Eq. 3 and Eq. 4.

- Noun-phrases' case insensitive precision and recall.

- P-norm (Eq. 7) function's output.

- Rescaled parser score defined in Eq. 8. Our first experiment will compare the correlation rate between metric using rescaled parser score and that using parser score directly.

\subsection{Different kernels}

Intuitively, features and the resulting assessment are not in a linear correlation. We trained two SVM, one with linear kernel and the other with Gaussian kernel, using NIST 2003 Chinese dataset. Then we apply the two metrics on NIST 2002 Chinese Evaluation dataset which has $3 \times 878=2634$ sentences ( 3 systems total). The results are summarized in Table 1. For comparison, the result from BLEU is also included.

\begin{tabular}{|c|c|c|c|}
\hline Feature & Linear & Gaussian & BLEU \\
\hline Rescale & 0.320 & $\mathbf{0 . 3 2 9}$ & \multirow{2}{*}{0.244} \\
\hline Direct & 0.317 & 0.224 & \\
\hline
\end{tabular}

Table 1: Spearman rank-correlation coefficients for regression based metrics using linear and Gaussian kernel, and using rescaled parser score or directly the parser score. Coefficient for BLEU is also involved.

Table 1 shows that the metric with Gaussian kernel using rescaled parser score gains the highest correlation rate. That is to say, Gaussian kernel function can capture characteristics of the relation better, and rescaling the parser score can help to increase the correlation with human judgments. Moreover, as other features range from 0 to 1 , we can discover in the second row of Table 1 that Gaussian kernel is suffering more seriously from the parser score which is ranging distinctly. In following experiments, we will adopt Gaussian kernel to train the SVM and rescaled parser score as a feature.

\subsection{Comparisons within the year 2003}

We held out 1/6 of the assessment dataset for parameter turning, and on the other $5 / 6$ of dataset, we perform a five-fold cross validation to verify the metric's performance. In comparison we introduce 
several metrics' coefficients reported in Albrecht and Hwa (2007) including smoothed BLEU (Lin and Och, 2004), METEOR (Banerjee and Lavie, 2005), HWCM (Liu and Gildea 2005), and the metric proposed in Albrecht and Hwa (2007) using the full feature set. The results are summarized in Table 2:

\begin{tabular}{|c|c|}
\hline Metric & Coefficient \\
\hline Our Metric & 0.515 \\
\hline Albrecht, 2007 & $\mathbf{0 . 5 2 0}$ \\
\hline Smoothed BLEU & 0.272 \\
\hline METEOR & 0.318 \\
\hline HWCM & 0.288 \\
\hline
\end{tabular}

Table 2: Comparison among various metrics. Learningbased metrics are developed from NIST 2003 Chinese Evaluation dataset and tested under five-fold cross validation.

Compared with reference based metrics such as BLEU, the regression based metrics yield a higher correlation rate. Generally speaking, for a given source sentence, there is usually a lot of feasible translations, but reference translations are always limited though this can be eased by adding references. On the other hand, regression based metrics is independent of references and make the assessment by mapping features to the score, so it can make a better judgment even dealing with a translation that doesn't match the reference well.

We can also see that our metric which uses only 6 features can reach a pretty high correlation rate which is close to the metric proposed in Albrecht and Hwa (2007) using 53 features. That confirms our speculation that a small feature set can also result in a metric having a good correlation with human judgments.

\subsection{Crossing years}

Though the training set and test set in the experiment described above are not overlapping, in the last, they come from the same dataset (NIST 2003). The content of this dataset are Xinhua news and AFC news from Jan. 2003 to Feb. 2003 which has an inherent correlation. To test the capability of generalization of our metric, we trained a metric on the whole NIST 2003 Chinese dataset $(20 \%$ data are held out for parameter tuning) and applied it onto NIST 2002 Chinese Evaluation dataset. We use the same metrics introduced in section 4.2 for comparison. The results are summarized in Table 3:

\begin{tabular}{|c|c|}
\hline Metric & Coefficient \\
\hline Our Metric & $\mathbf{0 . 3 2 9}$ \\
\hline Albrecht, 2007 & 0.309 \\
\hline Smoothed BLEU & 0.269 \\
\hline METEOR & 0.290 \\
\hline HWCM & 0.260 \\
\hline
\end{tabular}

Table 3: Cross year experiment result. All the learning based metrics are developed from NIST 2003.

The content of NIST 2002 Chinese dataset is Xinhua news and Zaobao's online news from Mar. 2002 to Apr. 2002. The most remarkable characteristic of news is its timeliness. News come from the year 2002 are nearly totally unrelated to that from the year 2003. It can be seen from Table 3 that we have got the expected results. Our metric can generalize well across years and yields a better correlation with human judgments.

\subsection{Discussions}

Albrecht and Hwa (2007) and this paper both adopted a regression-based learning method. In fact, the preliminary experiment is strictly set according to their paper. The most distinguishing difference is that the features in Albrecht and Hwa (2007) are collections of existing automatic evaluation metrics. The total 53 features are computationally heavy (for the features from METEOR, ROUGE, HWCM and STM). In comparison, our metric made use of six features coming from linguistic knowledge which can be easily obtained. Moreover, the experiments show that our metric can reach a correlation with human judgments nearly as good as the metric described in Albrecht and Hwa (2007), with a much lower computation cost. And when we applied it to a different year's dataset, its correlation rate is much better than that of the metric from Albrecht and Hwa (2007), showing us a good capability of generalization.

To account for this, we deem that the regression model is not resistant to data overfiting. If provided too much cross-dependent features for a limited training data, the model is prone to a less generalized result. But, it is difficult in practice to locate those key features in human perception of translation quality because we are lack of explicit evidences on what human actually use in translation evaluation. In such cases, this paper uses only "simple feature in key linguistic aspects", which reduces the risk of overfitting and bring a more generalized regression results. 
Compared with the literature, the "byte-length ratio between source and translation" and the "parse score" are original in automatic MT evaluation modeling. The parse score is proved to be a good alternative to LM. And it helps to avoid the errors of parser in parse structure (the experiment to verify this claim is still on-going).

It should be noted that feature selection is accomplished by empirically exhaustive test on the combination of the candidate features. In future work, we will test if this strategy will help to get better results for MT evaluation, e.g. try-on the selection between the 53 features in Albrecht and Hwa (2007). And, we will also test to see if linguistic motivated feature augmentation would bring further benefit.

\section{Conclusion}

For the metrics based on regressing, it is not always true that more features and complex features will help in performance. If we choose features elaborately, simple features are also effective. In this paper we proposed a regression based metric with a considerably small feature set that yield performance of the same level to the metrics with a large set of 53 features. And the experiment of the cross-year validation proves that our metric bring a more generalized evaluation results by correlating with human judgments better.

\section{Acknowledgements}

This research is support by Natural Science Foundation of China (Grant No. 60773066) and National 863 Project (Grant No. 2006AA01Z150)

\section{References}

Joshua S. Albrecht and Rebecca Hwa. 2007. A Reexamination of Machine Learning Approaches for Sentence-Level MT Evaluation. In Proceedings of the 45th Annual Meeting of the Association of Computational Linguistics, pages 880-887, Prague, Czech Republic, June.

Satanjeev Banerjee and Alon Lavie. 2005. METEOR: An Automatic Metric for MT Evaluation with Improved Correlation with Human Judgments. In Proceedings of the Workshop on Intrinsic and Extrinsic Evaluation Measures for MT and/or Summarization at the Association for Computational Linguistics Conference 2005: 65-73. Ann Arbor, Michigan.
John Blatz, Erin Fitzgerald, George Foster, Simona Gandrabur, Cyril Goutte, Alex Kulesza, Alberto Sanchis, and Nicola Ueffing. 2003. Confidence estimation for machine translation. In Technical Report Natural Language Engineering Workshop Final Report, pages 97-100, Johns Hopkins University.

Simon Corston-Oliver, Michael Gamon, and Chris Brockett. 2001. A machine learning approach to the automatic evaluation of machine translation. In Proceedings of the 39th Annual Meeting of the Association for Computational Linguistics, pages 140-147, Toulouse, France, July.

W. Gale and K. W. Church. 1991. A Program for Aligning Sentences in Bilingual Corpora. In Proceedings of the 29th Annual Meeting of the Association for Computational Linguistics, pages 177-184, Berkeley.

Jesús Giménez and Lluís Màrquez. 2007. Linguistic Features for Automatic Evaluation of Heterogenous MT Systems. In Proceedings of the Second Workshop on Statistical Machine Translation, pages 256264, Prague, Czech Republic, June.

Thorsten Joachims. 1999. Making large-scale SVM learning practical. In Bernhard Schöelkopf, Christopher Burges, and Alexander Smola, editors, $A d$ vances in Kernel Methods - Support Vector Learning. MIT Press.

Alex Kulesza and Stuart M. Shieber. 2004. A learning approach to improving sentence-level MT evaluation. In Proceedings of the 10th International Conference on Theoretical and Methodological Issues in Machine Translation (TMI), pages 75-84, Baltimore, MD, October.

Gregor Leusch, Nicola Ueffing, and Hermann Ney. 2006. CDER: Efficient MT evaluation using block movements. In The Proceedings of the Thirteenth Conference of the European Chapter of the Association for Computational Linguistics, pages 241-248.

Chin-Yew Lin \& Franz Josef Och. 2004. Automatic Evaluation of Machine Translation Quality Using Longest Common Subsequence and Skip-Bigram Statistics. In Proceedings of the 42nd Annual Meeting of the Association for Computational Linguistics, pages 606-613, Barcelona, Spain, July.

Ding Liu and Daniel Gildea. 2005. Syntactic features for evaluation of machine translation. In $A C L 2005$ Workshop on Intrinsic and Extrinsic Evaluation Measures for Machine Translation and/or Summarization, pages 25-32, June.

Christopher B. Quirk. 2004. Training a Sentence-Level Machine Translation Confidence Measure, In Proceedings of LREC 2004, pages 825-828.

Grazia Russo-Lassner, Jimmy Lin, and Philip Resnik. 2005. A Paraphrase-Based Approach to Machine Translation Evaluation. In Technical Report LAMPTR-125/CS-TR-4754/UMIACS-TR-2005-57, University of Maryland, College Park, August. 